\title{
Article \\ On the Performance of UAV-Aided Content Caching in Small-Cell Networks with Joint Transmission
}

\author{
Menghan Wei ${ }^{1}$, Youjia Chen ${ }^{1, *} \mathbb{D}$ and Ming Ding ${ }^{2}$ \\ 1 Fujian Key Lab for Intelligent Processing and Wireless Transmission of Media Information, College of Physics \\ and Information Engineering, Fuzhou University; N181127038@fzu.edu.cn \\ 2 Data 61, Commonwealth Scientific and Industrial Research Organisation (CSIRO), \\ Eveleigh, NSW 1435, Australia; Ming.Ding@data61.csiro.au \\ * Correspondence: youjia.chen@fzu.edu.cn
}

Citation: Wei, M.; Chen, Y.; Ding, M. On the Performance of UAV-Aided Content Caching in Small-Cell Networks with Joint Transmission. Electronics 2021, 10, 1040. https:// doi.org/10.3390/electronics10091040

Academic Editor: Nurul I. Sarkar

Received: 2 April 2021

Accepted: 25 April 2021

Published: 28 April 2021

Publisher's Note: MDPI stays neutral with regard to jurisdictional claims in published maps and institutional affiliations.

Copyright: (c) 2021 by the authors. Licensee MDPI, Basel, Switzerland. This article is an open access article distributed under the terms and conditions of the Creative Commons Attribution (CC BY) license (https:// creativecommons.org/licenses/by/ $4.0 /)$.

\begin{abstract}
Unmanned aerial vehicles (UAVs), featured by the high-mobility and high-quality propagation environment, have shown great potential in wireless communication applications. In this paper, a novel UAV-aided small-cell content caching network is proposed and analyzed, where joint transmission (JT) is considered in the dense small-cell networks and mobile UAVs are employed to shorten the serving distance. The system performance is evaluated in terms of the average cache hit probability and the ergodic transmission rate. From the analytical results, we find that (i) the proposed UAV-aided small-cell network shows superior caching performance and, even with a small density of UAVs the system's cache hit probability, can be improved significantly; (ii) the content's optimal caching probability to maximize the cache hit probability is proportional to the $(K+1)$-th root of its request probability, where $K$ is the number of small-cell base stations that serve each user by JT; (iii) caching the most popular content in UAVs may lead to a low transmission rate due to the limited resource offered by the low-density UAVs. Simulation results are presented to validate the theoretical results and the performance gain achieved by the optimal caching strategy.
\end{abstract}

Keywords: wireless caching; UAV-aided small-cell networks; joint transmission

\section{Introduction}

Caching popular content in dense-deployed small-cell base stations (SBSs) was proposed as a promising technology to reduce the transmission latency and relieve the backhaul congestion in 5G networks. The random caching strategy, which caches one file with a specific probability, was investigated in dense small-cell networks due to its low complexity [1]. Meanwhile, coordinated multi-point (CoMP) technologies enable the joint transmission (JT) of geographically separated BSs to serve a single user cooperatively, which shows a great potential of mitigating inter-cell interference and increasing the cell-edge users' performance, especially in dense small-cell networks [2].

To further improve caching performance and boost the caching utility, some works explored CoMP-JT to improve the caching performance. The CoMP among nearby SBSs was adopted in the small-cell caching network [3], and the diversity and multiplexing gains of caching were investigated. In [4], a combined caching scheme was proposed in a cluster-centric small-cell network. That is, the most popular content was cached in every SBS and delivered to users by joint transmission, while the less popular content was divided into different partitions, cached in multiple SBSs, and delivered by parallel transmission. Based on this combined caching model, the caching performance in terms of requesting successful probability and average transmission outage were investigated in [5].

Some works enabled both single transmission (ST) and JT in small-cell caching networks. In [6], the long-term-averaged SINR is introduced to identify the cell-core and cell-edge area. The users located in the cell-core area were served with ST, while the users in the cell-edge area could be served by JT to improve throughput. Compared with the 
deterministic network model adopted in [6], stochastic geometry was introduced into the caching analysis of large-scale networks. The homogeneous Poisson point process was used to model the distribution of SBSs in [7], and a dual-mode caching scheme was proposed and investigated. That is, the most popular files were stored at each SBS and served by JT, named cooperative plane, while less popular files were served by ST, named non-cooperative plane, to achieve spatial caching diversity.

Meanwhile, the advent and development of unmanned aerial vehicles (UAVs) have attracted plenty of attention in wireless applications, thanks to their mobility and flexible deployment, low deployment cost, line-of-sight (LoS) propagation of air-to-ground (A2G) links, etc. [8,9]. UAVs with caching functionality can efficiently ease the front-haul congestions, which was proposed and investigated recently in academia [10]. In [11], UAVs were utilized as content storage units that could transmit required files to users. Based on the prediction of user requests and mobility, a multi-objective optimization problem on user quality of experience and UAVs' transmission power was analyzed. In [12], multiple-input multiple-output (MIMO) technology was introduced into UAV networks to enhance the performance of random caching. In [13], the distributed beamforming in CoMP technologies was considered in UAV-enabled fog-RAN networks, where the average system capacity and energy efficiency were analyzed. Furthermore, the joint optimization of the UAVs distributed beamforming and locations was investigated to maximize the number of users who experienced a target QoS [14]. In [15], a hybrid caching strategy was investigated in UAV networks, where the contents in the popular set were multi-casted by geographically close UAVs and those in the unpopular set were transmitted from a single UAV. Moreover, a heterogeneous UAV network was considered in [16], where users could download files from the nearest UAV macro BSs or the UAV SBSs within a given radius.

Furthermore, UAV-mounted base stations were introduced to assist the terrestrial base stations. In [17], the UAVs were designed to fly at a fixed altitude following a circular trajectory with a certain radius centered at the ground SBSs to serve cell-edge users, while the SBSs focused on the cell-center users. The UAVs' trajectory and user partitioning were optimized to maximize the minimum throughput of all users. The UAVs were deployed to offer mmWave access for users who could not be served by macro cells due to blocking or interfering, where different backhauling modes were investigated [18]. In [19], the offloading from ground base stations to UAVs was analyzed, where UAVs hover at the typical location of the cell center in a quasi-static manner as a second layer to serve a portion of the users. The UAV altitude and its transmission power were optimized due to the co-channel interference.

Recently, the caching performance of heterogeneous networks consisting of UAVmounted base stations and ground base stations was also analyzed. In [20], cache-enabled UAVs were considered to assist a macro base station with limited backhaul capacity, where the mobility of UAVs was utilized to enhance the system performance. In [21], UAVs served the users within a predetermined distance and the user associated with ground base stations if the requested file could be achieved from UAVs. In [22], UAVs equipped with storage capacity act complementary to the base stations for offloading traffic from the cellular network. Under the constraint of the UAV's limited battery, the UAV placement, caching, and user association were jointly optimized to maximize the cache hit ratio. Furthermore, in [23], the cooperation among the UAVs within a cylinder was considered in such heterogeneous network. In [24], a co-channel deployment of UAVs and SBSs was considered, and the user was served by a UAV or an SBS based on its maximum received power. Then, the successful content delivery probability and energy efficiency were derived based on the association probabilities with UAVs and SBSs.

Considering the ultra-dense small-cell network deployed in 5G and the limited UAV density due to power limitation and strong LoS interference, in this paper, we aim to explore an efficient way to utilize the UAVs to enhance the caching performance in small cells. Hence, a novel UAV-aided small-cell caching network is proposed and analyzed, where the dense SBSs work as the majority of wireless caching while the UAVs play an 
auxiliary role. Moreover, the JT among SBSs is considered to fully explore the potential of the ultra-dense small-cell network, and the mobility of UAVs is utilized to improve the signal transmission. The contributions of this paper are summarized as follows.

- The cache hit probability is derived, from which a significant enhancement can be observed compared with the tranditional small-cell networks with ST, contributed by the good SINR from the JT among SBSs and the good propagation environment of UAVs. It is notable that a very low density of UAVs could bring a significant improvement, which makes this scheme more practical.

- The content transmission rate is also derived, where the different resource allocated to users served by JT and single transmission (ST) is considered. An adjustable parameter based on SNR is introduced to control the radius of ST, which shows a tradeoff between cache hit probability and content transmission rate. Moreover, the optimal caching strategy is investigated in the scenario where each user is served by $K$ SBSs. The content's optimal caching probability is obtained through an approximated closed-form of the cache hit probability, which is proportional to the $(K+1)$-th root of the request probability.

- A high density of UAVs will in fact degrade the cache hit probability, due to the severe interference caused by UAVs' good transmission conditions. On the other hand, considering the limited radio resource used by low-density UAVs, caching the top popular files may lead to an over-loaded situation.

\section{System Model}

\subsection{Network Model}

In the considered heterogeneous wireless caching network consisting of SBSs and UAVs, as illustrated in Figure 1, the distributions of SBSs, UAVs and users are modeled as independent homogeneous Poisson Point Processes (HPPPs) in a plane, denoted by $\Phi$, $\Psi$ and $\Xi$, respectively. (Aiming at more tractable results and hence valuable insights, the PPP model is adopted in this work. More delicate stochastic geometry models or realistic models driven by real-life experiments will be considered as our future work.) Considering the height disparity between UAVs and terrestrial SBSs or users, we assume that the UAVs are deployed in a vertical height $H$, while the vertical height of the SBSs and users are assumed to be 0 .

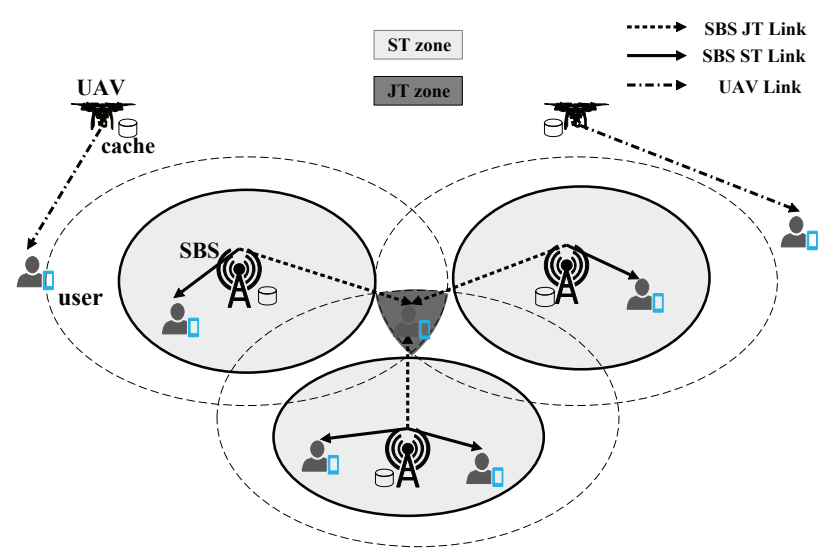

Figure 1. Illustration of UAV-aided small-cell network.

$\lambda_{\mathrm{b}}, \lambda_{\mathrm{v}}$ and $\lambda_{\mathrm{u}}$ denote the densities for the SBSs, UAVs and users, respectively. Due to the implementation of dense SBSs and the energy constraint of UAVs, UAVs are relatively sparsely deployed compared with ground SBSs, i.e., $\lambda_{\mathrm{v}} \ll \lambda_{\mathrm{b}}$. Moreover, the transmission power of SBSs and UAVs are denoted by $P_{\mathrm{b}}$ and $P_{\mathrm{v}}$, respectively.

Importantly, it is assumed that SBSs and UAVs are operated on different frequency spectra avoid the severe interference at SBSs caused by UAVs due to their good propagation condition [23]. Furthermore, as shown in [24], the co-channel deployment of UAVs and 
SBSs makes almost $90 \%$ of users associate with UAVs even if the UAV density is only $\frac{1}{9}$ of the SBS density. The allocation of different spectra for UAVs and SBSs can prevent this kind of load unbalance and fully utilize the resources of high-density ground SBSs. Since the interference co-ordination schemes such as power control may be a useful tool to mitigate these problem, it shall be considered in our future work.

The list of system notations and their descriptions have been summarized in Table 1.

Table 1. Summary of notations.

\begin{tabular}{ll}
\hline Notation & Description \\
\hline$\Phi, \Psi, \Xi$ & set of SBSs, UAVs and users \\
$\lambda_{b}, \lambda_{v}, \lambda_{u}$ & density of SBSs, UAVs and users \\
$H$ & UAV height \\
$P_{b}, P_{v}$ & transmission power of SBSs and UAVs \\
$\alpha_{b}, \alpha_{v}$ & path loss exponent of SBSs and UAVs \\
$q_{f}$ & request probability of the $f$-th file \\
$c_{f}^{b}, c_{f}^{v}$ & caching probability of the $f$-th file in SBSs and UAVs \\
$\Phi_{f}, \Psi_{f}$ & set of SBSs and UAVs caching the $f$-th file \\
$\Xi_{f}$ & set of users requesting the $f$-th file \\
$\rho$ & the parameter for adjusting the range of JT \\
$D$ & the UAV flight horizontal distance for hovering \\
$T$ & user's maximum waiting time \\
$V$ & the flying speed of UAVs \\
$\kappa$ & the users within this distance will be served by ST \\
$\delta$ & the minimum SINR requirement for data decoding \\
$\Gamma_{f}^{b}, \Gamma_{f}^{v}$ & received SINR from SBSs and UAVs in Slice- $f$ \\
$\zeta_{f}^{b}, \zeta_{f}^{v}$ & cache hit probability achieved from SBSs and UAVs in Slice- $f$ \\
$N_{f}^{b}, N_{f}^{v}$ & the number of users in an SBS cell and a UAV cell in Slice- $f$ \\
$B_{b}, B_{v}$ & available bandwidth in an SBS and a UAV \\
$R_{f}^{b}, R_{f}^{v}$ & the average transmission rate achieved from SBSs and UAVs in Slice- $f$ \\
$\zeta$ & the average cache hit probability in this network \\
$R$ & the average transmission rate in this network \\
\hline
\end{tabular}

\subsection{Channel Model}

The path loss between an SBS and a user with a horizontal distance $r$, i.e., an SBS-user link, is modeled as

$$
\mathrm{f}_{b}(r)=A_{b} r^{-\alpha_{b}},
$$

where $A_{b}$ denotes the path loss at a reference distance and $\alpha_{b}$ denotes the path loss exponent.

In contrast, considering the vertical height of UAVs, the distance between a UAV and a user with a horizontal distance $r$ is given as $\sqrt{r^{2}+H^{2}}$. Hence, the pass loss function for a UAV-user link is formulated as

$$
\mathfrak{ł}_{v}(r)=A_{v}\left(\sqrt{r^{2}+H^{2}}\right)^{-\alpha_{v}},
$$

where $A_{v}$ and $\alpha_{v}$ represent the corresponding path loss parameters for UAVs. Due to the high probability of line-of-sight transmission path for UAV-user links, usually, we have $\alpha_{v}<\alpha_{b}$ [25].

The multi-path fading between SBSs or UAVs and users is assumed to be independently and identically Rayleigh-distributed. Hence, the fading power is a unit-mean exponential random variable $h$, where we have $h \sim \exp (1)$.

\subsection{Caching Model}

Let us consider a file library consisting of $F$ different files, where the request probability for the $f$-th file is denoted by $q_{f}$, satisfying $0 \leq q_{f} \leq 1, \forall f \in[1, \cdots, F]$ and $\sum_{f=1}^{F} q_{f}=1$. 
Without loss of generality, we assume that $q_{1} \geq q_{2} \geq \ldots \geq q_{F}$ and that, at a particular time instant, a user can only request one file. According to the independent thinning theory, the distribution of users who request the $f$-th file in one time instant can be viewed as an HPPP with the density of $q_{f} \lambda_{u}$.

On the other hand, following the probabilistic caching strategy, each file is cached with a specific caching probability. That is, the $f$-th file is randomly cached in SBSs and $\mathrm{UAV}$ s with the probability $c_{f}^{b}$ and $c_{f}^{v}$, respectively. Moreover, the maximum number of files cached in SBSs and UAVs are denoted by $N$ and $M$, respectively. Similarly, the SBSs and UAVs caching the $f$-th file can be viewed as two independent thinned HPPPs with the densities $c_{f}^{b} \lambda_{b}$ and $c_{f}^{v} \lambda_{v}$, respectively.

Aiming to conveniently analyze the network performance related to the $f$-th file, we have the following definition.

Definition of Slice- $f$ : The sets of SBSs and UAVs caching the $f$-th file, and the users requesting the $f$-th file in the network, are defined as Slice- $f$, which includes the HPPPs $\Phi_{f}, \Psi_{f}$ and $\Xi_{f}$, with the densities $c_{f}^{b} \lambda_{b}, c_{f}^{v} \lambda_{v}$ and $q_{f} \lambda_{u}$, respectively.

\section{The Proposed Service Scheme}

As mentioned before, a UAV-aided small-cell caching scheme is adopted in this work. Firstly, in this network, the SBSs act as the major caching servers for content delivery due to their dense deployment, sufficient power supply, stable backhaul capacity and convenient cache update. Secondly, BS-enabled UAVs work as assistants of SBSs in content caching and serving. The main reason for this design is that a dense deployment of BS-enabled UAVs leads to a poor downlink performance due to the severe interference caused by UAVs' good propagation environment [26]. Considering the limited resource provided by such low-density UAVs, UAVs play an auxiliary role in content serving. Only when a user cannot obtain its requested file from SBSs do they turn to UAVs for help.

\subsection{SBS Joint Transmission Scheme}

We consider the JT among SBSs aiming at fully exploring the potential of densedeployed SBSs. The JT scheme leads to a larger received signal but is quit demanding in implementation. For the JT strategy, both channel state information and user data should be shared by all cooperating BSs, and thus each user receives data from multiple BSs, which requires a tight synchronization among the involved BSs. In practice, cooperating SBSs are connected through imperfect backhaul links with finite capacity, unpredictable latency, and limited connectivity. These limitations mean that if a cooperating SBS fails to achieve tight synchronization with the other cooperative BSs or fails to obtain both the transmission data and the CSI in time, then it has to quit the JT operations. Thus, an adjustable parameter $\rho$ is introduced to determine whether a user is served by JT. That is, only when the user's received SNR from its nearest SBS caching the requested file is less than a predetermined threshold, $\rho$ will the user will be served in the JT way. That is,

$$
\text { user associates } K \operatorname{SBS}(s):\left\{\begin{array}{l}
K=1, \quad \text { if } \operatorname{SNR}\left(r_{1}\right)>\rho, \\
K>1, \quad \text { otherwise, }
\end{array}\right.
$$

where $r_{1}$ is the distance between the user and its nearest SBS caching that requested file.

\subsection{UAV Serving Scheme}

The mobility of UAVs is utilized to improve the caching performance. That is, the UAV will fly towards the user to shorten the serving distance and hence improve the wireless signal $[20,27]$. It is assumed that UAVs are flying at the same height to follow the government guide and to avoid a potential collision. Furthermore, hence, the drone collision issue can be addressed within the cellular infrastructure, which does not require coordination among UAVs from different business sectors, such as BS-enabled UAVs, delivery UAVs, traffic surveillance UAVs, etc. A horizontal distance $D$ is chosen to decide 
whether the UAV needs to fly. In more detail, if the horizontal distance between the UAV and its serving user is larger than $D$, then UAV would fly towards the user. Otherwise, it will hover at its current position.

Moreover, a maximum time for user waiting, $T$, is set to restrict the maximum flying time of UAVs. To save users' waiting time, it is assumed that UAVs will fly with a high speed; hence, the power consumption in the flying model will be larger than that in the hovering model. Hence, to save energy and waiting time, when the transmission distance is reduced to $D$, the UAV will stop moving and switch to hovering. Note that the trade-off between the UAV energy consumption and the achieved average transmission rate can be adjusted by the value of $D$. The optimal value of $D$ is also an important performance factor of UAV caching networks, which is one of our future research directions. Note that, we assume that the UAV will not transmit files during flying to avoid instability.

$r$ denotes the horizontal distance between the serving UAV and the user, and $V$ the UAV flying speed. Based on the above flying scheme, there are three outcomes of the horizontal serving distance, $r^{\prime}$, as illustrated in Figure 2:

- $\quad r>V T+D$ : After the flying with the maximum time $T$, the horizontal distance between the UAV and the user is $r^{\prime}=r-V T$, which is still larger than $D$.

- $\quad D<r \leq V T+D$ : Within the time $T$, the UAV reaches the predetermined distance $D$. Hence, $r^{\prime}=D$.

- $\quad r \leq D$ : Since the horizontal distance is less than $D$, the UAV directly transmits data to the user without extra flying. Hence, $r^{\prime}=r$.
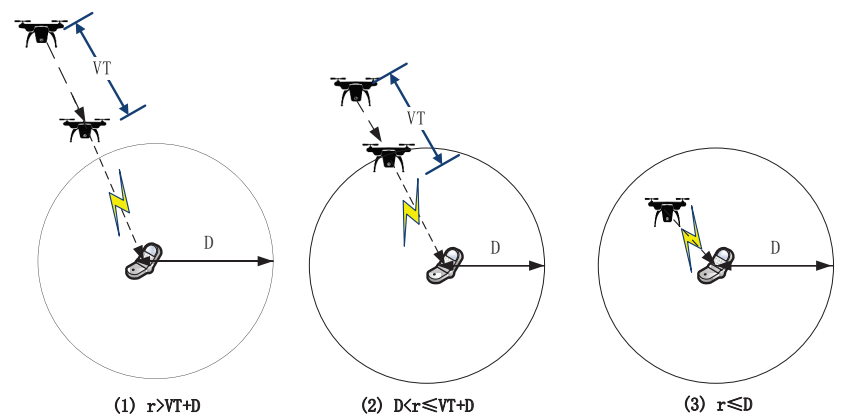

Figure 2. Three cases of UAV's flight chart.

In Sections 4 and 5, we analyze the caching performance from SBSs and UAVs. Without loss of generality, the caching performance in Slice- $f$ is derived with the assumption that a typical user in Slice- $f$ is located at the origin.

\section{Analysis on Cached Small-Cells with Joint Transmissions}

\subsection{SINR from K SBSs with JT Scheme}

According to the user association criterion in Equation (3) and the path loss in Equation (2), the SNR from the nearest SBS in slice- $f$ can be formulated as $\frac{P_{\mathrm{b}} A_{\mathrm{b}} r_{1}^{-\alpha_{\mathrm{b}}}}{\sigma^{2}}$, where $\sigma^{2}$ denotes the Gaussian noise power. (According to [28], the average received signal is used to determine the association between users and SBSs. Hence, the small-scale fading is averaged in the SNR for association.) Hence, given $\rho$, this association criterion implies that users within the disk of $\kappa$ are served by ST, i.e., $r_{1}<\kappa$, where $\kappa=\left(\frac{P_{b} A_{b}}{\rho \sigma^{2}}\right)^{\frac{1}{\alpha_{b}}}$. 
When the typical user is locates outside this disk, it is served by the JT of $K$ nearest SBSs, and its received SINR can be formulated as follows.

$$
\begin{aligned}
& \Gamma_{f}^{b}(\mathbf{r})=\frac{S_{b}}{I_{b}+\sigma^{2}}=\frac{\sum_{k=1}^{\mathrm{K}} P_{b} A_{b} r_{k}^{-\alpha_{b}} h_{k}}{I_{b 1}+I_{b 2}+\sigma^{2}} \\
& =\frac{\sum_{k=1}^{\mathrm{K}} P_{b} A_{b} r_{k}^{-\alpha_{b}} h_{k}}{\sum_{y_{i} \in \Phi_{f} \backslash \mathbf{x}} P_{P_{b}} A_{b}\left\|y_{i}\right\|^{-\alpha_{b}} h_{y_{i}}+\sum_{y_{j} \in \Phi \backslash \Phi_{f}} P_{b} A_{b}\left\|y_{j}\right\|^{-\alpha_{b}} h_{y_{j}}+\sigma^{2}},
\end{aligned}
$$

where the received signal power $S_{b}$ is the sum of the received signal power coming from the $K$ serving SBSs, $\mathbf{x}$. The aggregated interference comes from two parts: (i) the SBSs in the same slice but not the serving ones, $\Phi_{f} \backslash \mathbf{x}$, i.e., the SBSs caching the target file but further than the $K$-th nearest SBS, and (ii) the SBSs belonging to other slices, $\Phi \backslash \Phi_{f} . \mathbf{r}$ denotes the distance from the $K$ serving SBSs; that is, $\mathbf{r}=\left\{r_{1}, \ldots, r_{k}, \ldots, r_{K}\right\}$, where $1 \leq k \leq K$, denotes the vector of serving distance. Due to the HPPP of $\Phi_{f}$, the joint probability density function (PDF) of $\mathbf{r}$ can be formulated as

$$
f_{f}^{b}(\mathbf{r}) \triangleq f\left(r_{1}, \cdots, r_{K}\right)=\exp \left(-\pi c_{f}^{b} \lambda_{b} r_{K}^{2}\right)\left(2 \pi c_{f}^{b} \lambda_{b}\right) \prod_{k=1}^{K} r_{k} .
$$

\subsection{Cache Hit Probability Achieved from Cached SBSs}

Cache hit probability is a commonly used performance metric in caching networks, which measures the probability that the users can successfully download their requested content from pre-cached BSs. In wireless works, the event of cache hit can be evaluated by the event that the received SINR from the cached SBS is larger than a decoding threshold [29]. Hence, the cache hit probability that the typical user in Slice- $f$ achieves from SBSs can be formulated as

$$
\begin{aligned}
\zeta_{f}^{b} \triangleq & \operatorname{Pr}\left[\Gamma_{f}^{b}>\delta\right] \\
& \stackrel{(a)}{=} \int_{0}^{\mathcal{K}} \operatorname{Pr}\left[\Gamma_{f}^{b}\left(r_{1}\right)>\delta\right] f_{f}^{b}\left(r_{1}\right) \mathrm{d} r_{1}+ \\
& \int_{\mathcal{K}}^{\infty} \int_{r_{2}<\cdots<r_{K}} \operatorname{Pr}\left[\Gamma_{f}^{b}(\mathbf{r})>\delta\right] f_{f}^{b}(\mathbf{r}) \prod_{k=1}^{K} \mathrm{~d} r_{k},
\end{aligned}
$$

where $\delta$ denotes the minimum SINR requirement for data decoding and $(a)$ considers the JT scheme mentioned in Section 3.1.

In the above equation, given the serving-distance vector, $\mathbf{r}$, the probability $\operatorname{Pr}\left[\Gamma_{f}^{b}(\mathbf{r})>\delta\right]$ can be derived as:

$$
\begin{aligned}
& \operatorname{Pr}\left[\Gamma_{f}^{b}(\mathbf{r})>\delta\right]=\operatorname{Pr}\left[\frac{S_{b}}{I_{b}+\sigma^{2}}>\delta\right] \\
& \stackrel{(a)}{=} \int_{0}^{\frac{S_{b}}{\delta}-\frac{\sigma^{2}}{P_{b} A_{b}}} \frac{1}{2 \pi j} \int_{-j \infty}^{+j \infty} \mathcal{L}_{I_{b 1}}(z) \mathcal{L}_{I_{b 2}}(z) \exp (-z x) \mathrm{d} z \mathrm{~d} x \\
& =\int_{-j \infty}^{+j \infty} \frac{1-\exp \left(-\frac{z}{\delta} S_{b}\right) \exp \left(\frac{z \sigma^{2}}{P_{b} A_{b}}\right)}{2 \pi j z} \mathcal{L}_{I_{b 1}}(z) \mathcal{L}_{I_{b 2}}(z) \mathrm{d} z,
\end{aligned}
$$


where $(a)$ uses the characteristic function [30] and $\mathcal{L}_{I}(\cdot)$ denotes the Laplace transform of I. Moreover, the three components are given by

$$
\begin{gathered}
\exp \left(-\frac{z}{\delta} S_{b}\right)=\prod_{k=1}^{\mathrm{K}} \mathbb{E}_{h_{k}}\left[\exp \left(-\frac{z}{\delta} h_{k} r_{k}^{-\alpha_{b}}\right)\right] \\
=\prod_{k=1}^{\mathrm{K}} \frac{1}{1+\frac{z}{\delta} r_{k}^{-\alpha_{b}}}, \\
\mathcal{L}_{I_{b 1}}(z)=\mathbb{E}_{h_{y_{i}}, y_{i}}\left[\exp \left(-z \sum_{y_{i} \in \Phi_{f} \backslash x} h_{y_{i}}\left\|y_{i}\right\|^{-\alpha_{b}}\right)\right] \\
=\exp \left(-2 \pi c_{f}^{b} \lambda_{b} \frac{z r_{K}^{2-\alpha_{b}}}{\alpha_{b}-2}{ }_{2} \mathrm{~F}_{1}\left(1,1-\frac{2}{\alpha_{b}} ; 2-\frac{2}{\alpha_{b}} ;-\frac{z}{r_{K}^{\alpha_{b}}}\right)\right) \\
\triangleq \exp \left(-2 \pi c_{f}^{b} \lambda_{b} r_{K}^{2} \cdot A\left(\frac{z}{r_{K}^{\alpha_{b}}}, \alpha_{b}\right)\right), \\
\mathcal{L}_{I_{b 2}}(z)=\mathbb{E}_{h_{y_{j}}, y_{j}}\left[\exp \left(-z \sum_{y_{j} \in \Phi \backslash \Phi_{f}} h_{y_{j}}|| y_{j} \|^{-\alpha_{b}}\right)\right] \\
=\exp \left(-2 \pi\left(1-c_{f}^{b}\right) \lambda_{b} \frac{z^{\frac{2}{\alpha_{b}}}}{\alpha_{b}} B\left(\frac{2}{\alpha_{b}}, 1-\frac{2}{\alpha_{b}}\right)\right) \\
\triangleq \exp \left(-2 \pi\left(1-c_{f}^{b}\right) \lambda_{b} C\left(z, \alpha_{b}\right)\right) .
\end{gathered}
$$

Here, ${ }_{2} \mathrm{~F}_{1}(\cdot)$ denotes the hypergeometric function, $B(\cdot)$ represents the beta function [31], and $A(x, y) \triangleq \frac{x}{y-2} 2 \mathrm{~F}_{1}\left(1,1-\frac{2}{y} ; 2-\frac{2}{y} ;-x\right), C(x, y) \triangleq \frac{x^{\frac{2}{y}}}{y} B\left(\frac{2}{y}, 1-\frac{2}{y}\right)$.

\subsection{Transmission Rate Achieved from Cached SBSs}

Apparently, the achievable transmission rate highly depends on the allocated timefrequency resource. Due to the JT scheme introduced before, users in the network can be divided into two groups: the users served by JT and the users served by ST. Compared with the users served by ST, who only occupy the time-frequency resource on one SBS, the users served by JT occupy the resource on all the $K$ serving SBSs.

Assuming that an SBS equally allocates its resources to the associated users, JT and ST ones, the resource allocated for one JT user from the $K$ SBSs could serve $K$ ST users. Hence, an equivalent user density is introduced to approximately calculate the density of resource-sharing "users" in the network, that is,

$$
\operatorname{Pr}[\mathcal{A}] \lambda_{u}+K \operatorname{Pr}[\overline{\mathcal{A}}] \lambda_{u}
$$

where event $\mathcal{A}$ denotes a user is served by ST, while $\overline{\mathcal{A}}$ denotes a user is served by JT.

\subsubsection{Average Number of Users Associated with an SBS}

For the typical user in Slice- $f$, the probability that it is served by ST, event $\mathcal{A}_{f}$, can be calculated by the probability that its SNR is larger than $\rho$, which means $r_{1}<\kappa$. That is,

$$
\begin{aligned}
\operatorname{Pr}\left[\mathcal{A}_{f}\right] & =\int_{0}^{\left(\frac{P_{b} A_{b}}{\rho \sigma^{2}}\right)^{\frac{1}{\alpha_{b}}}} f_{f}^{b}\left(r_{1}\right) \mathrm{d} r_{1} \\
& =1-\exp \left(-\pi c_{f}^{b} \lambda_{b}\left(\frac{P_{b} A_{b}}{\rho \sigma^{2}}\right)^{\frac{2}{\alpha_{b}}}\right) .
\end{aligned}
$$


Hence, the equivalent user density in Slice- $f$ is given by:

$$
\operatorname{Pr}\left[\mathcal{A}_{f}\right] q_{f} \lambda_{u}+K \operatorname{Pr}\left[\overline{\mathcal{A}_{f}}\right] q_{f} \lambda_{u}
$$

$N_{f}^{b}$ denotes the number of users associated with an SBS in Slice- $f$, according to [32], we have that $N_{f}^{b}$ approximately follows a Negative Binomial distribution, that is,

$$
N_{f}^{b} \sim \mathrm{NB}\left(Q, \frac{q_{f} \lambda_{u}\left(\operatorname{Pr}\left[\mathcal{A}_{f}\right]+K \operatorname{Pr}\left[\overline{\mathcal{A}_{f}}\right]\right)}{q_{f} \lambda_{u}\left(\operatorname{Pr}\left[\mathcal{A}_{f}\right]+K \operatorname{Pr}\left[\overline{\mathcal{A}_{f}}\right]\right)+c_{f}^{b} \lambda_{b}}\right) .
$$

\subsubsection{Transmission Rate from SBSs}

$B_{b}$ denotes the available spectrum resource in an SBS, and the bandwidth allocated for a user served by ST in Slice- $f$ equals to $\frac{B_{b}}{N_{f}^{b}}$. For JT, which requires the same spectrum allocated to the user in all the $K$ SBSs, the bandwidth allocated to a JT user depends on the user number in the cooperative $K$ SBSs. For tractable results, we use the average number of users to approximate the user number in each SBS, and hence, the bandwidth allocated to a user served by JT is $\frac{K B_{b}}{N_{f}^{b}}$.

Considering the JT scheme with the adjustable parameter $\rho$, two cases need to be investigated: (i) the typical user in Slice- $f$ is served by ST, i.e., located inside the disk of $\kappa$, and (ii) the typical user is served by JT of $K$ SBSs. Hence, we can formulate the average transmission rate of the typical user in Slice- $f$ as

$$
\begin{gathered}
R_{f}^{b}=\mathbb{E}\left[\frac{B_{b}}{N_{f}^{b}}\right] \int_{0}^{\mathcal{K}} \mathbb{E}\left[\log _{2}\left(1+\Gamma_{f}^{b}\left(r_{1}\right)\right)\right] f_{f}^{b}\left(r_{1}\right) \mathrm{d} r_{1} \\
+\frac{B_{b}}{\mathbb{E}\left[N_{f}^{b}\right]} \int_{\mathcal{K}}^{\infty} \int_{r_{2}<\ldots<r_{k}}^{\mathbb{E}}\left[\log _{2}\left(1+\Gamma_{f}^{b}(\mathbf{r})\right)\right] f_{f}^{b}(\mathbf{r}) \mathrm{d} \mathbf{r},
\end{gathered}
$$

where $\mathbb{E}\left[\log _{2}\left(1+\Gamma_{f}^{b}\right)\right]$ can be derived as:

$$
\begin{aligned}
& \mathbb{E}\left[\log _{2}\left(1+\Gamma_{f}^{b}\right)\right]=\int_{\delta}^{\infty} \log _{2}(1+\gamma) f_{\Gamma_{f}^{b}}(\gamma) \mathrm{d} \gamma \\
= & \int_{\delta}^{\infty} \frac{\operatorname{Pr}\left[\Gamma_{f}^{b}>\gamma\right]}{(1+\gamma) \ln 2} \mathrm{~d} \gamma+\log _{2}(1+\delta) \operatorname{Pr}\left[\Gamma_{f}^{b}>\delta\right],
\end{aligned}
$$

where $\delta$ is the minimum SINR requirement for data decoding, and $\operatorname{Pr}\left[\Gamma_{f}^{b}>\gamma\right]$ is computed by $(7)$.

\section{Analysis on Cached UAVs}

\subsection{SINR from $U A V$}

Given the horizontal serving distance, $r^{\prime}$, the received SINR of the typical user in Slice- $f$ served by a UAV can be formulated as

$$
\begin{aligned}
& \Gamma_{f}^{v}=\frac{S_{v}}{I_{v}+\sigma^{2}}=\frac{P_{v} A_{v}\left(r^{\prime 2}+H^{2}\right)^{-\frac{\alpha_{v}}{2}} h}{I_{v 1}+I_{v 2}+\sigma^{2}} \\
& =\frac{P_{v} A_{v}\left(r^{\prime 2}+H^{2}\right)^{-\frac{\alpha_{v}}{2}} h}{\sum_{y_{i} \in \Psi_{f} \backslash x} P_{v} A_{v}\left(\left\|y_{i}\right\|^{2}+H^{2}\right)^{-\frac{\alpha v v}{2}} h_{y_{i}}+\sum P_{v} P_{v}\left(\left\|y_{j}\right\|^{2}+H^{2}\right)^{-\frac{\alpha_{v}}{2}} h_{y_{j}}+\sigma^{2}},
\end{aligned}
$$

where the received signal power $S_{v}$ comes from the nearest UAV in Slice- $f$. The aggregate interference consists of two parts similar to $I_{b}$ in Equation (4). In the above derivation, it is 
assumed that the distribution of $\mathrm{UAVs}^{\prime}$ locations with movement can be approximated as a PPP according to Monte-Carlo simulations.

\subsection{Cache Hit Probability Achieved Using Cached UAVs}

Lemma 1. Given the horizontal distance between the serving UAV and the user, $r$, and its corresponding horizontal serving distance, $r^{\prime}$, the probability $\operatorname{Pr}\left[\Gamma_{f}^{v}\left(r^{\prime}, r\right)>\delta\right]$ can be calculated as

$$
\begin{aligned}
& \operatorname{Pr}\left[\Gamma_{f}^{v}\left(r^{\prime}, r\right)>\delta\right]=\exp \left(-\frac{\left(r^{\prime 2}+H^{2}\right)^{\frac{\alpha_{v}}{2}} \delta \sigma^{2}}{A_{v} P_{v}}\right) \\
& \cdot \exp \left(-2 \pi c_{f}^{v} \lambda_{v}\left(r^{2}+H^{2}\right) \cdot A\left(\delta\left(\frac{r^{\prime 2}+H^{2}}{r^{2}+H^{2}}\right)^{\frac{\alpha_{v}}{2}}, \alpha_{v}\right)\right) \\
& \cdot \exp \left(-2 \pi\left(1-c_{f}^{v}\right) \lambda_{v} H^{2} \cdot A\left(\delta\left(\frac{r^{\prime 2}+H^{2}}{H^{2}}\right)^{\frac{\alpha_{v}}{2}}, \alpha_{v}\right)\right) .
\end{aligned}
$$

Then, considering the three cases of horizontal serving distance mentioned in Section 3.2, we can formulate the cache hit probability achieved using a cached UAV in Slice- $f$ as

$$
\begin{aligned}
\zeta_{f}^{v} & \triangleq \operatorname{Pr}\left[\Gamma_{f}^{v}>\delta\right]=\int_{0}^{D} \operatorname{Pr}\left[\Gamma_{f}^{v}(r, r)>\delta\right] f_{f}^{v}(r) \mathrm{d} r \\
& +\int_{D}^{V T+D} \operatorname{Pr}\left[\Gamma_{f}^{v}(D, r)>\delta\right] f_{f}^{v}(r) \mathrm{d} r \\
& +\int_{V T+D}^{\infty} \operatorname{Pr}\left[\Gamma_{f}^{v}(r-V T, r)>\delta\right] f_{f}^{v}(r) \mathrm{d} r
\end{aligned}
$$

where the PDF $f_{f}^{v}(r)=\exp \left(-\pi c_{f}^{v} \lambda_{v} r^{2}\right) \cdot 2 \pi c_{f}^{v} \lambda_{v} r$.

\subsection{Transmission Rate Achieved Using Cached UAV}

According to the designed file-download scheme, only when a user fails to get the requested file from SBSs will they turn to UAVs for file download. Hence, in Slice- $f$, the density of UAVs is $c_{f}^{v} \lambda_{v}$, and the density of users served by UAVs is given by $\left(1-\zeta_{f}^{b}\right) q_{f} \lambda_{u}$. The number of users associated with one UAV in Slice- $f, N_{f}^{v}$, is given as

$$
N_{f}^{v} \sim \mathrm{NB}\left(Q, \frac{\left(1-\zeta_{f}^{b}\right) q_{f} \lambda_{u}}{\left(1-\zeta_{f}^{b}\right) q_{f} \lambda_{u}+c_{f}^{v} \lambda_{v}}\right) .
$$

Under the assumption of equal resource allocation among users, considering the three cases of UAV transmission in Figure 2, the average transmission rate of the typical user in Slice- $f$ achieved from the UAV can be formulated as

$$
\begin{aligned}
& R_{f}^{v}=\mathbb{E}\left[\frac{B_{v}}{N_{f}^{v}}\right] \int_{0}^{D} \mathbb{E}\left[\log _{2}\left(1+\Gamma_{f}^{v}(r, r)\right)\right] f_{f}^{v}(r) \mathrm{d} r \\
& +\mathbb{E}\left[\frac{B_{v}}{N_{f}^{v}}\right] \int_{D}^{D+V T} \mathbb{E}\left[\log _{2}\left(1+\Gamma_{f}^{v}(D, r)\right)\right] f_{f}^{v}(r) \mathrm{d} r \\
& +\mathbb{E}\left[\frac{B_{v}}{N_{f}^{v}}\right] \int_{D+V T}^{\infty} \mathbb{E}\left[\log _{2}\left(1+\Gamma_{f}^{v}(r-V T, r)\right)\right] f_{f}^{v}(r) \mathrm{d} r
\end{aligned}
$$

where $B_{v}$ denotes the available spectrum resource in one $\mathrm{UAV}$, and the bandwidth allocated for each user is given as $\frac{B_{v}}{N_{f}^{v}}$. Furthermore, $N_{f}^{v}$ distribution is given in Equation (20). The following calculating steps are similar to SBSs referred to Equation (16), which are omitted here for brevity. 


\section{Performance of UAV-Aided Small-Cell Caching Networks and Optimization}

In the above sections, we derive the cache hit probability and the transmission rate achieved from cache-enabled SBSs and UAVs separately. In this section, we formulate the overall performance of this UAV-aided small-cell caching network. Moreover, the optimal caching schemes in SBSs and UAVs are discussed. Due to the complexity of the formulation, two special cases are studied.

\subsection{Caching Performance Achieved}

\subsubsection{Average Cache Hit Probability}

As mentioned in Section 3, the SBSs act as the major caching servers for content delivery, where a user will turn to UAVs for requested content only when it cannot obtain the content from SBSs.

Based on the results of the cache hit probability achieved by SBSs and UAVs in Slice- $f$, i.e., $\zeta_{f}^{b}$ in Equation (6) and $\zeta_{f}^{v}$ in Equation (19), and the content download scheme, the average cache hit probability of the proposed UAV-aided small-cell caching system, $\zeta$, is given by:

$$
\zeta=\sum_{f=1}^{F} q_{f} \cdot\left[\zeta_{f}^{b}+\left(1-\zeta_{f}^{b}\right) \zeta_{f}^{v}\right]
$$

\subsubsection{Ergodic Transmission Rate}

Moreover, the ergodic transmission rate that a user can achieve when downloading the requested content is written as

$$
R=\sum_{f=1}^{F} q_{f} \cdot\left[\zeta_{f}^{b} R_{f}^{b}+\left(1-\zeta_{f}^{b}\right) R_{f}^{v}\right]
$$

where $R_{f}^{b}$ and $R_{f}^{v}$ have been derived in Equations (15) and (21).

\subsection{Discussion on Optimal Caching Strategy}

To maximize the utility of content caching, in the following, we investigate the optimal caching probability of each file to maximize the cache hit probability [33]. Furthermore, considering the requirement of content delivery, such as video streaming, the transmission rate needs to satisfy a minimum requirement. Hence, we have the following optimization problem.

$$
\begin{array}{ll} 
& \underset{\left(c_{f}^{b}, c_{f}^{v}\right)}{\operatorname{maximize} \zeta} \\
\text { s. t. } \quad & 0 \leq c_{f}^{b} \leq 1, \quad 0 \leq c_{f}^{v} \leq 1 ; \\
& \sum_{f=1}^{F} c_{f}^{b}=N, \quad \sum_{f=1}^{F} c_{f}^{v}=M ; \\
& R_{f}^{b}>R_{\min }, \quad R_{f}^{v}>R_{\min } ;
\end{array}
$$

where Constraint (24b) guarantees that the caching probability for each file is in the feasible region of $[0,1]$; Constraint (24c) considers the maximum storage of SBSs and UAVs, i.e., SBSs can cache at most $N$ files and UAVs can cache at most $M$ files; and Constraint (24d) represents the minimum requirement of transmission rate, denoted by $R_{\min }$.

The optimal caching strategy consists of the file caching PMF in SBSs and in UAVs, i.e., $c_{f}^{b(o p t)}$ and $c_{f}^{v(o p t)}$. Since a user will try to get their request file from SBSs first and turn to UAVs only when such action fails, we discuss this optimal caching strategy separately 
in two parts: (i) $c_{f}^{b(o p t)}$ to maximize the average cache hit probability of SBSs, $\zeta^{b}$, and (ii) $c_{f}^{v(o p t)}$ to maximize the average cache hit probability of UAVs, $\zeta^{v}$.

\subsubsection{Optimal Caching Strategy in SBSs}

Firstly, we focus on the objective of maximizing the cache hit probability from SBSs, i.e., $\zeta^{b}=\sum_{f=1}^{F} q_{f} \zeta_{f}^{b}$. Due to the complexity of the formulation $\zeta_{f}^{b}$ in Equation (6), the optimization problem is extremely challenging.

To obtain the close-form expression $\zeta^{b}$, we consider a special case, where each user in the network is served by JT of $K$ SBSs, i.e., $\rho=\infty$, and the interference-limited network with the noise $\sigma^{2}=0$. Moreover, the $K$ times of the signal from the $K$-th nearest SBS is adopted to approximate the lower bound of the signal strength.

Lemma 2. Given $\rho=\infty$ and $\sigma^{2}=0$, the lower bound of the cache hit probability, $\widetilde{\zeta^{b}}$, can be approximated as

$$
\widetilde{\zeta^{b}} \approx \sum_{f=1}^{F} q_{f}\left(\frac{c_{f}^{b}}{2 c_{f}^{b} A\left(\frac{1}{K} \delta, \alpha_{b}\right)+2\left(1-c_{f}^{b}\right) C\left(\frac{1}{K} \delta, \alpha_{b}\right)+c_{f}^{b}}\right)^{K},
$$

where $C\left(\frac{1}{K} \delta, \alpha_{b}\right)=\frac{1}{\alpha_{b}}\left(\frac{1}{K} \delta\right)^{\frac{2}{\alpha_{b}}} B\left(\frac{2}{\alpha_{b}}, 1-\frac{2}{\alpha_{b}}\right)$.

Proof of Lemma 2. Consider the interference-limited network, i.e., $\sigma^{2}=0$, the SIR is approximated by the $K$ times of the signal from the $K$-th nearest SBS, which can be formulated as

$$
\begin{aligned}
\Gamma_{f}^{b} & \approx \frac{K P_{b} A_{b} r_{K}^{-\alpha_{b}} h_{K}}{I_{b 1}+I_{b 2}} \\
& =\frac{K P_{b} A_{b} r_{K}^{-\alpha_{b}} h_{K}}{\sum_{y_{i} \in \Phi_{f} \backslash\{\mathbf{x}\}} P_{b} A_{b}\left\|y_{i}\right\|^{-\alpha_{b}} h_{y_{i}} \sum_{y_{j} \in \Phi \backslash \Phi_{f}} P_{b} A_{b}\left\|y_{j}\right\|^{-\alpha_{b}} h_{y_{j}}} .
\end{aligned}
$$

Hence, we have

$$
\begin{aligned}
& \widetilde{\zeta^{b}}=\int_{0}^{\infty} \int_{r_{2}<\ldots<r_{k}} \operatorname{Pr}\left[\frac{K P_{b} A_{b} r_{K}^{-\alpha_{b}} h_{K}}{I_{b 1}+I_{b 2}} \geq \delta\right] f_{f}^{b}(\mathbf{r}) \prod_{k=1}^{K} \mathrm{~d} r_{k} \\
& =\int_{0}^{\infty} \int_{r_{2}<\ldots<r_{K}} \mathcal{L}_{I_{b 1}}\left[-\frac{1}{K} r_{K}^{\alpha_{b}} \delta\right] \mathcal{L}_{I_{b 2}}\left[-\frac{1}{K} r_{K}^{\alpha_{b}} \delta\right] \\
& \exp \left(-\pi c_{f}^{b} \lambda_{b} r_{K}^{2}\right)\left(2 \pi c_{f}^{b} \lambda_{b}\right)^{K} r_{1} \ldots r_{K} \mathrm{~d} r_{K} \ldots r_{1},
\end{aligned}
$$

where $\mathcal{L}_{I_{b 1}}\left[-\frac{1}{K} r_{K}^{\alpha_{b}} \delta\right]$ and $\mathcal{L}_{I_{b 2}}\left[-\frac{1}{K} r_{K}^{\alpha_{b}} \delta\right]$ have been derived in Equations (9) and (10) with $z=-\frac{1}{K} r_{K}^{\alpha_{b}} \delta$.

Then we can have the following optimization problem:

$$
\begin{gathered}
\underset{c_{f}^{b}}{\operatorname{maximize}} \widetilde{\zeta^{b}}, \\
\text { s.t. } \quad 0 \leq c_{f}^{b} \leq 1, \\
\\
\sum_{f=1}^{F} c_{f}^{b}=N .
\end{gathered}
$$


Due to the high density of SBSs in current and future cellular networks and the sufficient spectrum resource provided, we assume that once the received SINR is larger than the threshold $\delta$, the transmission rate can meet the requirement. Hence, the constraint about transmission rate is neglected in the above optimization problem. For this optimization problem, we have the following theorem.

Theorem 1. The optimal caching strategy, $c_{f}^{b(o p t)}$, to maximize $\widetilde{\zeta^{b}}$ can be solved by convex optimization theory, which is given as

$$
c_{f}^{b(o p t)}=\min \left\{\left\lceil\frac{\sqrt[K+1]{\frac{q_{f}}{\zeta}}-2 C\left(\frac{1}{K} \delta, \alpha_{b}\right)}{2 A\left(\frac{1}{R} \delta, \alpha_{b}\right)-2 C\left(\frac{1}{R} \delta, \alpha_{b}\right)+1}\right\rceil^{+}, 1\right\},
$$

where

$$
\sqrt[K+1]{\bar{\xi}}=\frac{\sum_{f=1}^{F^{*}} \sqrt[K+1]{q_{f}}}{2\left(F^{*}-N\right) C\left(\frac{1}{K} \delta, \alpha_{b}\right)+2 N A\left(\frac{1}{K} \delta, \alpha_{b}\right)+N},
$$

and $F^{*}, 1 \leq F^{*} \leq F$ satisfies the constraint that $c_{f}^{b} \geq 0, \forall f$.

From the above optimal result, we can see that in the scenario of JT with K SBSs, a file's optimal caching probability is proportional to the $(K+1)$-th root of its request probability. Hence, a more popular file will have a larger caching probability; however, this disparity in caching probabilities is mitigated by the increasing number of SBSs cooperated in a JT scheme. This is because of the fact that the increase of signal power in JT enhances the received SINR and hence the cache hit probability_in such cases, the profit of caching the most popular files to enhance the average cache hit probability-will be less.

\subsubsection{Optimal Caching Strategy in UAVs}

Different from the dense SBSs, UAVs have a much smaller deployment density. Considering their large coverage and limited spectrum resource, the file transmission rate in Constraint (24d) cannot be neglected in the UAV caching scheme. In other words, the caching scheme should ensure the UAVs are not overloaded with the wireless traffic.

For tractable results, we consider a special case where $T=0$ and $H=0$. (The performance impact of the UAVs height is very limited in the scenarios with the typical flight height $H \in[30 \mathrm{~m}, 60 \mathrm{~m}]$ and the typical UAV density $\lambda_{v} \in\left[1 / \mathrm{km}^{2}, 10 / \mathrm{km}^{2}\right]$. Figure 3 illustrates $\mathrm{UAV}$ average cache hit probability $\zeta^{v}$ versus flight height $H$ in different $\lambda_{v}$, which confirms $\zeta^{v}$ is not sensitive to $H$.) Similar to the derivation steps in Proof of Lemma 2, we have the following optimization problem:

$$
\begin{gathered}
\max _{c_{f}^{v}} \sum_{f=1}^{F} \frac{q_{f} \cdot c_{f}^{v}}{2 c_{f}^{v} A\left(\delta, \alpha_{v}\right)+2\left(1-c_{f}^{v}\right) C\left(\delta, \alpha_{v}\right)+c_{f}^{v}} . \\
\text { s.t. } \quad 0 \leq c_{f}^{v} \leq 1, \\
\sum_{f=1}^{F} c_{f}^{v}=M, \\
R_{f}^{v}>R_{\min } .
\end{gathered}
$$


Theorem 2. The optimal caching strategy in UAVs, $c_{f}^{v(o p t)}$, to maximize the UAV's cache hit probability, is given as

$$
c_{f}^{v(o p t)}=\min \left\{\left[\frac{\sqrt{\frac{q_{f}}{\xi^{\prime}}}-2 C\left(\delta, \alpha_{v}\right)}{2 A\left(\delta, \alpha_{v}\right)-2 C\left(\delta, \alpha_{v}\right)+1}\right]^{+}, 1\right\}, f \in[G \ldots F],
$$

where

$$
\sqrt{\tilde{\xi}^{\prime}}=\frac{\sum_{f=G}^{F^{*}} \sqrt{q_{f}}}{2\left(F^{*}-M-G+1\right) C\left(\delta, \alpha_{v}\right)+2 M A\left(\delta, \alpha_{v}\right)+M^{\prime}}
$$

and $G=\min \left\{f: q_{f}<\frac{B_{v} \log _{2}(1+\delta) c_{f}^{v} \lambda_{v}}{R_{\min }\left(1-\zeta_{f}^{b}\right) \lambda_{u}}\right\}, G \leq F^{*} \leq F$.

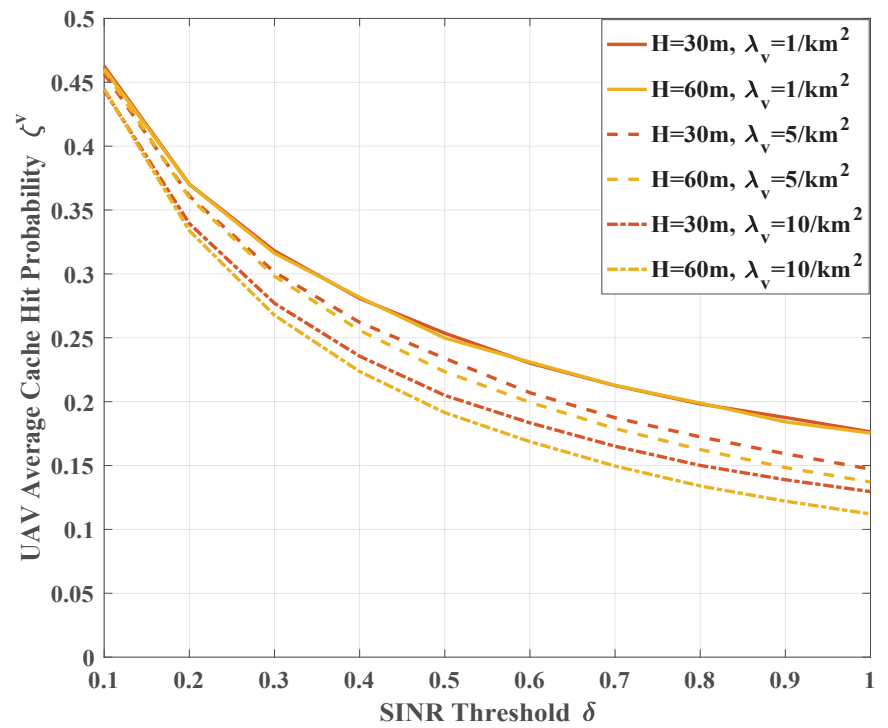

Figure 3. UAV average cache hit probability with different flight height $H$.

Compared with the optimal caching probability in SBSs, we can see that the most popular files may be excluded in UAVs, because a large number of requesting users may lead to very limited resources for each user, and hence a small transmission rate, which is a requirement that is hard to meet.

\section{Simulation Results}

In this section, we present the numerical results obtained by the analysis and the corresponding Monte-Carlo simulation results for various scenarios. In the Monte-Carlo simulation, the performance is averaged over 2000 network deployments in an area of $3.5 \times 3.5 \mathrm{~km}^{2}$. Moreover, the densities of users, deployed SBSs and UAVs, are $500 / \mathrm{km}^{2}$, $80 / \mathrm{km}^{2}$ and $4 / \mathrm{km}^{2}$, respectively. The pass loss exponents for non-line-of-sight transmission dominated SBSs and line-of-sight transmission dominated UAVs are set to $\alpha_{b}=4$ and $\alpha_{v}=2.9$, respectively. The SINR threshold is set to $0 \mathrm{~dB}$. The adjustable parameter for JT scheme $\rho$ based on SNR is chosen as $24 \mathrm{~dB}$ [34]. The predetermined serving distance threshold of UAV D and the UAV flying speed $V$ are set to $250 \mathrm{~m}$ and $30 \mathrm{~m} / \mathrm{s}$, respectively, considering the maximum waiting time of user $T$ as $2 \mathrm{~s}$. Hence, the maximum flight distance can be computed as $V \cdot T=60 \mathrm{~m}$. For simplicity, we consider the scenario of JT with $K=2$ SBSs, and we focus on the spectrum efficiency of SBSs and UAVs, neglecting their bandwidth. 
We consider the caching probability of the f-th file equals to its request probability, i.e., $c_{f}=q_{f}$, where $q_{f}$ follows the Zipf distribution, i.e, $q_{f}=\frac{\frac{1}{f^{\beta}}}{\sum_{i=1}^{F} \frac{1}{i^{\beta}}}$, where $\beta$ is the skewing factor and set to 1 in the simulations. We consider a file library consisting of 100 different files, and the maximum storage in SBSs and UAVs is $N=20$ and $M=10$, respectively.

\subsection{Performance Improvement of $U A V$-aided}

In Figure 4, we plot the caching performance, in terms of the average cache hit probability, with different caching schemes: (1) small-cell caching with ST scheme, (2) small-cell caching with JT scheme, (3) UAV-aided small-cell caching with ST scheme and (4) UAV-aided small-cell caching with JT scheme. Firstly, it can be seen that the numerical results closely match the simulation results for all schemes, which validates the accuracy of our theoretical result. Secondly, the JT scheme among SBSs outperforms the ST one, which is not surprising. When $\rho=24 \mathrm{~dB}$, the performance gain of the average cache hit probability of SBS, $\zeta^{b}$, reaches $30 \%-50 \%$ with different SINR thresholds. More importantly, the assisted UAVs with an extremely low density, less than one-tenth of SBS density, bring a notable performance improvement. Based on the ST scheme, UAVs bring almost more than $40 \%$ gain in terms of cache hit probability and based on the JT scheme, the performance gain still approaches $20 \%$.

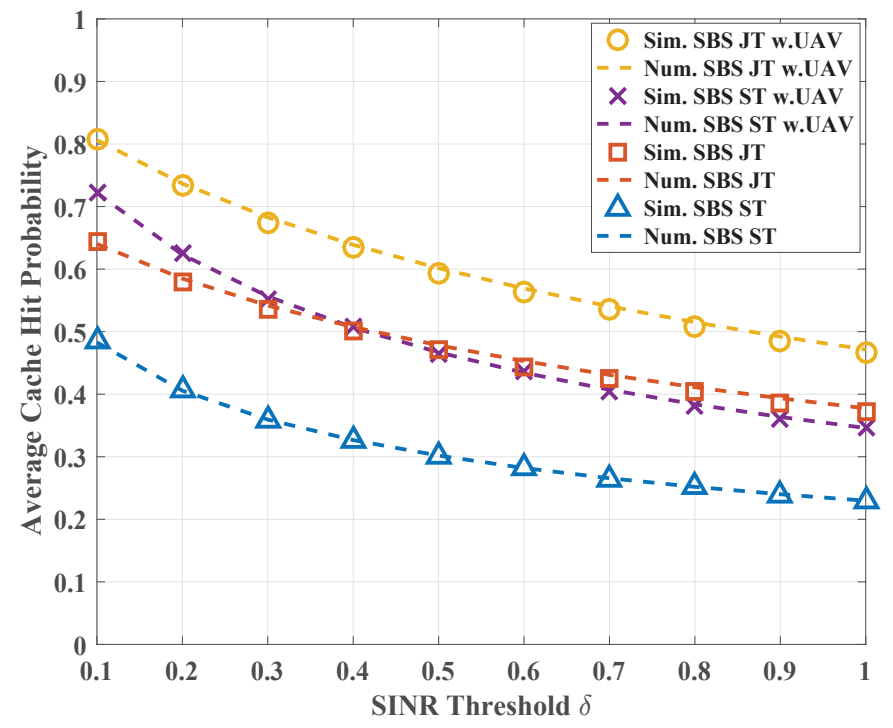

Figure 4. Comparison of cache hit probability of SBS ST scheme, SBS JT scheme and UAV assisted SBS JT scheme.

\subsection{Performance of SBS Caching with JT Scheme}

Figure 5 plots the average cache hit probability of SBSs, $\zeta^{b}$, versus different adjustable parameters of the JT strategy, $\rho$. Meanwhile, different SBS densities $\lambda_{b}$ and Zipf exponents $\beta$ for file request distribution are also investigated. From the figure, we can see that:

- $\quad$ The average cache hit probability $\zeta^{b}$ increases with the growth of $\rho$ in all the scenarios. As $\rho$ increases, more users will be served by JT, which results in a stronger received signal power and hence an enhancement of caching performance, $\zeta^{b}$.

- Interestingly, from the figure, increasing the density of SBSs does not bring any performance improvement but degrades the cache hit probability in many scenarios, which is counterintuitive. The reason behind this phenomenon lies in the ST range determined by the adjustable parameter $\rho$. As the SBS density $\lambda_{b}$ grows, the probability of users located inside the range $\kappa$ and using ST increase, as shown in Equation (12). Hence, from the perspective of the cache hit probability, the lower averaged SINR performance due to fewer users served by JT leads to the degradation of $\zeta^{b}$. 
- Moreover, we can see that the impacts of the adjustable parameter $\rho$ and the SBS density $\lambda_{b}$ are more obvious with a larger Zipf exponent. As we have known, a larger exponent means a more unevenly distributed file request probability. With the default caching strategy, $c_{f}=q_{f}$, the more uneven one yields a more skewed cache hit probability due to the larger weight of several popular files. On the other hand, this disparity of weights will enlarge the performance impact brought by other factors.

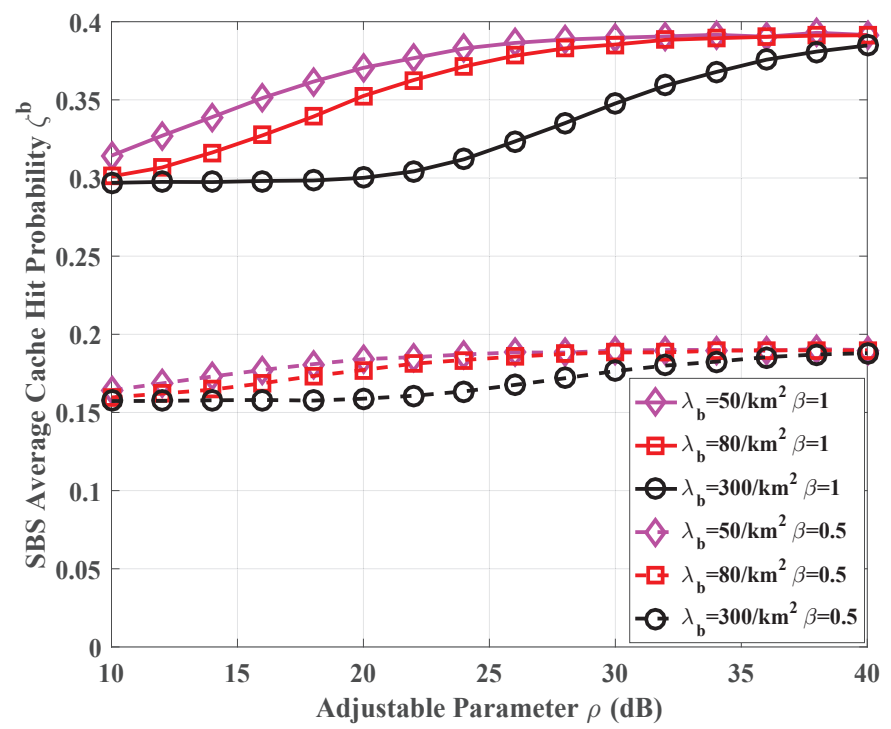

Figure 5. Cache hit probability of SBSs vs. the adjustable parameter for JT.

As mentioned in previous paragraphs, the increase in SBS density does not lead to improvement of the cache hit probability, due to the fewer JT operations controlled by the adjustable parameter $\rho$. In Figure 6, we tend to show the caching benefits obtained by the network densification; specifically, there is a trade-off in the JT scheme.

- Firstly, from the figure, we can see that although the average cache hit probability decreases as the SBS density $\lambda_{b}$ grows, the average transmission rate increases with the increase in $\lambda_{b}$. The main reason lies in the more spectrum resources per area, although the SINR performance of users not served by JT degrades due to the growth of aggregate interference. The fewer users served by one SBS, the more bandwidth offered for each user.

- Secondly, both the cache hit probability and the transmission rate with the adjustable parameter $\rho=30 \mathrm{~dB}$ are better than that with $\rho=24 \mathrm{~dB}$. With a smaller ST range set by $30 \mathrm{~dB}$, more users can enjoy the JT operation, which results in the improvement of SINR. Note that the more users served by JT, the more cost in backhaul and signaling due to the operation complexity of JT.

- Thirdly, to balance the performance of the user's cache hit probability and transmission rate achieved, the density of SBSs has to be carefully chosen. If the SBS density is extremely high, a careful adjustment of $\rho$ is another way to avoid the decline of the cache hit probability. 


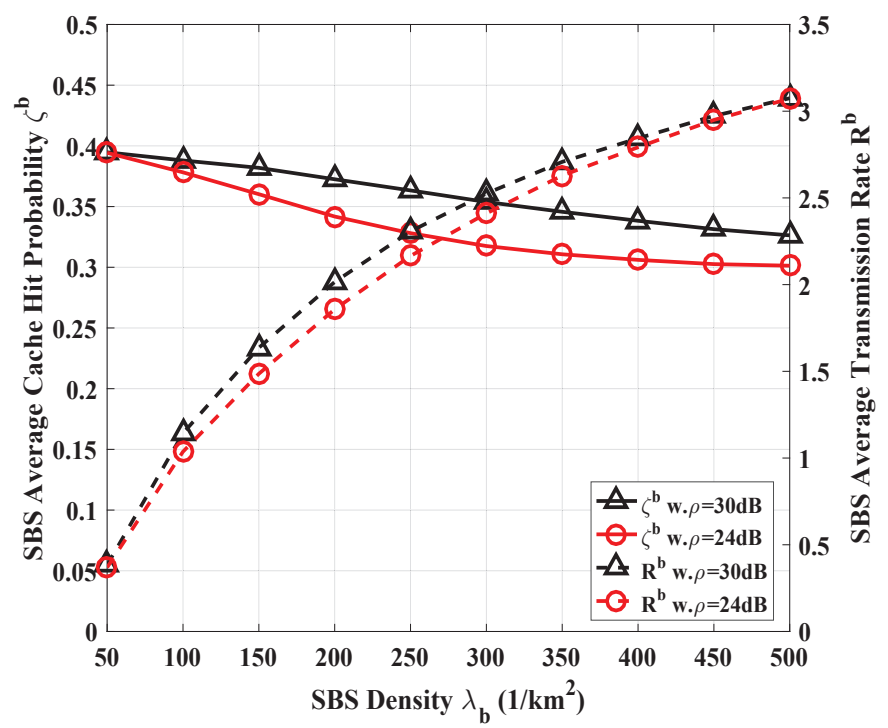

Figure 6. Averaged cache hit probability and transmission rate of SBSs.

Figure 7 aims to show the performance impact of the adjustable parameter $\rho$ on the average transmission rate of SBSs, $R^{b}$, in different scenarios with different user densities. From Figure 6, one might think that a larger $\rho$ always leads to a larger probability of JT and hence a larger transmission rate. From Figure 7, we will find that such a conclusion is conditional. In more detail,

- Generally speaking, all the curves share a similar trend: the increase in $R^{b}$ comes after a slight decrease regime. As $\rho$ grows, the ST range $\kappa$ declines. In this process, the users located at the cell edge are served by JT at the beginning, then the users at a medium distance, and last, the users near the cell center join in as well. Note that the users served by JT will receive more spectrum resources than ST users. Compared with cellcenter users, the cell-edge users have worse SINR performance due to long-serving distance. Therefore, the more spectrum resource of the network allocated to these poor-SINR users, the worse overall rate of the network, although the performance of these users is enhanced and the fairness is improved. This is the reason for the decreasing regime of average transmission rate.

- More importantly, we can see from the figure that the enhancement of the average transmission rate brought by the growth of adjustable parameter $\rho$ is not notable, especially in the scenarios with low user density or SBS density. However, it should be noted that the growth of $\rho$ leads to more users served by a JT scheme with a higher operational expense.

- A larger user density leads to a smaller piece of resource allocated to each user, and results in a lower average transmission rate for the typical user. Meanwhile, a larger SBS density means more spectrum resources per area $\lambda_{b} B$ can be used, and hence a better performance of the network. 


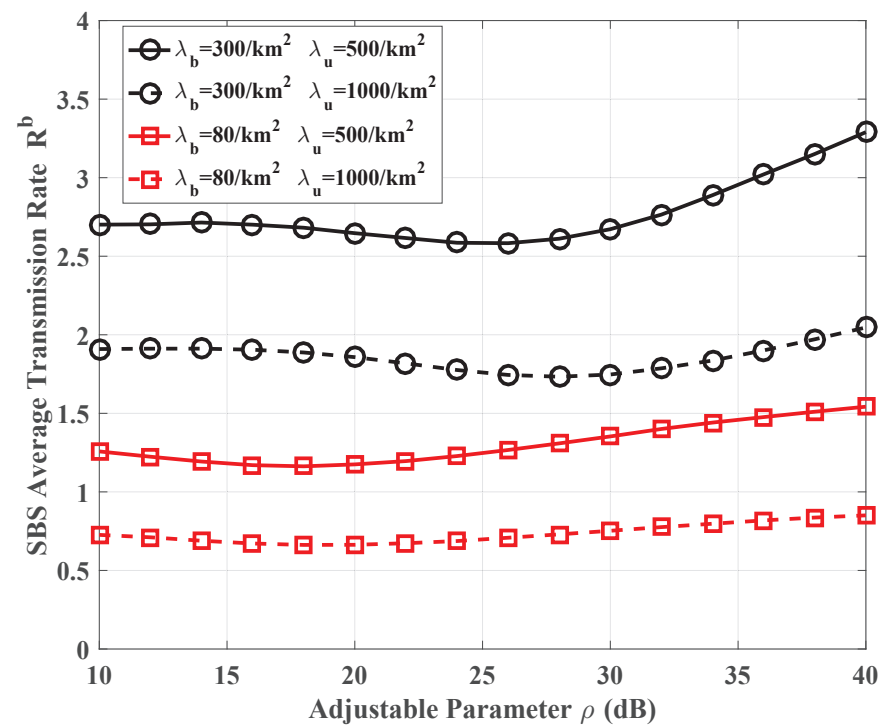

Figure 7. Average transmission rate of SBSs with different user densities vs. the adjustable parameter $\rho$.

\subsection{Performance Analysis of UAV Caching}

Figure 8 plots the average cache hit probability of UAVs, $\zeta^{v}$, versus different UAVs densities $\lambda_{v}$, and also shows the performance impact of UAVs' the predetermined serving distance threshold $D$ and the maximum flight distance $V$.

- Firstly, with the increase in the UAVs' density, the average cache hit probability $\zeta^{0}$ generally decreases. The reason is the good propagation environment of UAVs. That is, when the UAVs become denser, the aggregated interference grows even faster than the signal power due to the slowly decaying interference caused by the good propagation environment. Similar results have also been reported in [26].

- Secondly, from the figure, it can be observed that the smaller value of $D$, the better cache hit probability. Apparently, it contributes to a shorter average transmission distance, which enhances the received signal power and hence the caching hit probability.

- Thirdly, a larger maximum flight distance of UAVs, $V T$, yields a better caching performance of UAVs. However, it is notable that the increase of this value means a longer waiting time for users.

Figure 9 plots the average transmission rate when downloading files from UAVs, $R^{v}$, versus UAVs densities with different caching schemes: (1) the baseline caching PMF i.e., $c_{f}=q_{f}$; all caching (2) the most popular file; (3) the second popular one and (4) the third popular one. From the figure, we can see that

- In contrast to the average cache hit probability, the average transmission rate benefits from the increasing UAV density. The denser the UAVs deployed, the more radio resources offered. Furthermore, a higher transmission rate can hence be achieved, similar to the conclusions for small-cells.

- The baseline caching PMF has the worst rate performance compared with the strategies choosing popular files. Obviously, this tells us that the caching strategy should be optimized in order to achieve a good performance.

- Most importantly, from the figure, comparing the transmission rate achieved by caching the most popular file, the second popular file and the third popular file, we found that caching the most popular file performs worst, whose average transmission rate is much smaller than the other two. The reason behind this is that the low-density UAVs offer very limited radio resources, and hence it is hard to support many users. Note that a more popular file attracts more users.

- According to the previous analysis, UAVs caching the most popular files will greatly improve the cache hit probability, but from this figure, the transmission rate will 
degrade severely. Therefore, the cache scheme of UAVs should be carefully chosen in order to provide a sufficient transmission rate to users. Caching the most popular one might not be a good choice, especially for the low-density UAVs.

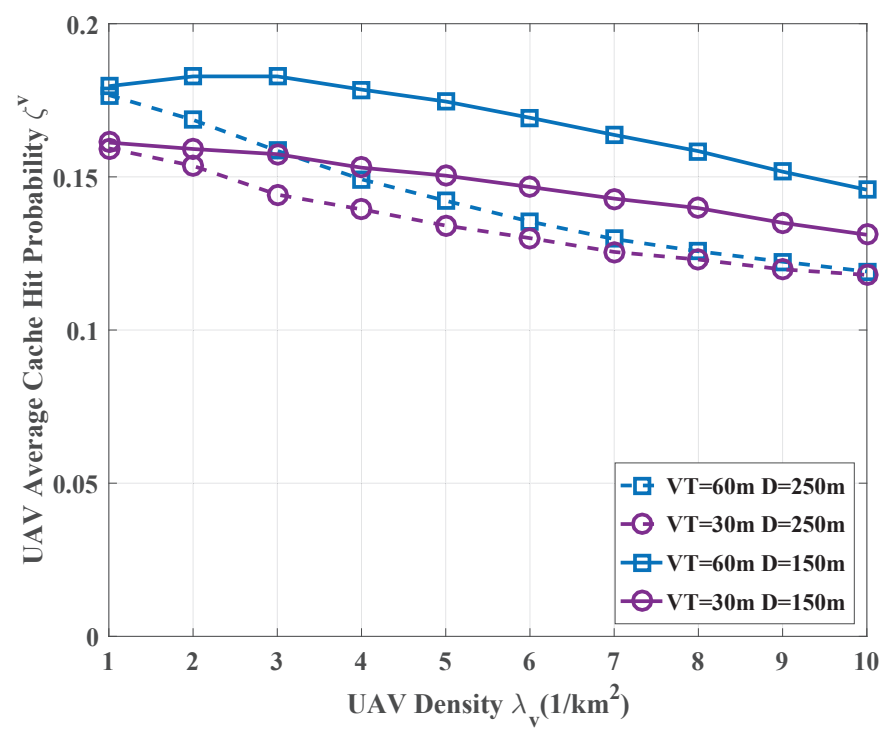

Figure 8. Average cache hit probability vs. UAV density for UAV with different the predetermined serving distance threshold or the maximum flight distance.

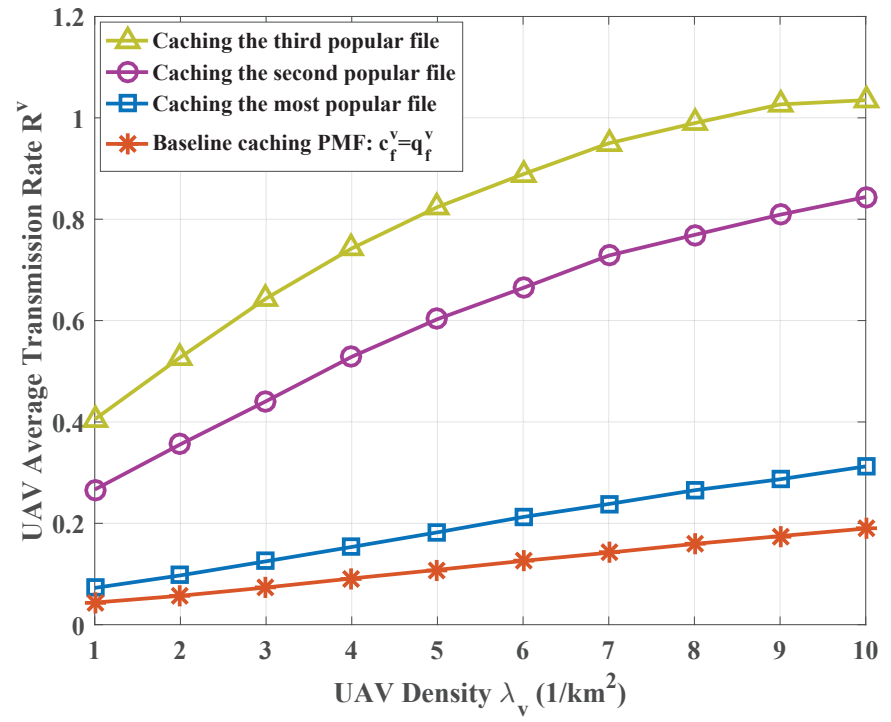

Figure 9. Average transmission rate vs. UAV density for UAV with different the predetermined serving distance threshold or the maximum flight distance.

\subsection{Caching Performance with Optimized Caching Strategies}

In Figure 10, we compare the average cache hit probability, $\zeta$, achieved by our proposed strategy with other two different strategies: (1) the linear caching probability $c_{f}=q_{f}$ and (2) the hybrid caching strategies proposed in [24]. From the figure, we can observe that:

- Compared with the other two caching strategies, our proposed optimal caching strategies for SBSs and UAVs can improve the average cache hit probability. Furthermore, this improvement is more obvious in the scenarios where a larger SINR threshold is required.

- In the medium-SINR region, the performance gains in SBSs, $\zeta^{b}$, achieved by the proposed optimal caching PMF, i.e., Equation (29), is near 20\% and 10\% compared to 
the linear caching strategy and the strategy in [24], respectively. The gains on the UAV part, $\zeta^{v}$, achieved by Equation (32), is around 30\% and 20\% compared to the linear caching strategy and the strategy in [24], respectively. As a result, the overall gain in terms of $\zeta$ approaches $25 \%$ and $8 \%$, compared with the two benchmark caching strategies.

- In the high-SINR region, the performance gains of $\zeta^{b}$ achieved by the proposed optimal caching PMF, i.e., Equation (29), is near 25\% and 11\% compared to the linear caching strategy and the strategy in [24], respectively. The gains of $\zeta^{v}$, achieved by Equation (32), is approximately equal to the medium SNR region. As a result, the overall gain in terms of $\zeta$ approaches $30 \%$ and $10 \%$, compared with the two benchmark caching strategies.

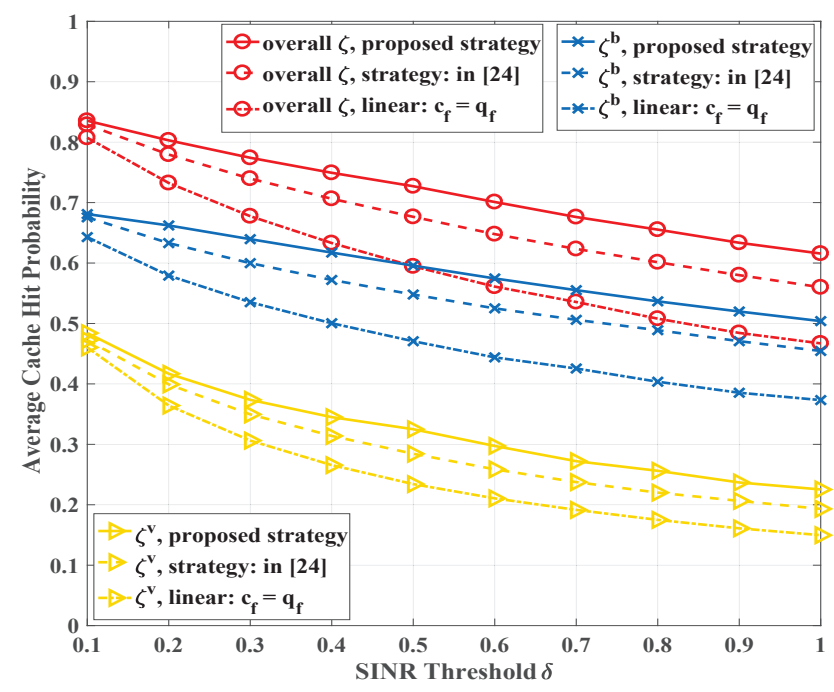

Figure 10. Comparison of average cache hit probability of two caching strategies.

\section{Conclusions}

In this paper, the caching performance in a UAV-aided small-cell network is analyzed in terms of the cache hit probability and transmission rate. The JT scheme is considered for the high-dense small-cell networks, and the mobility of UAVs is exploited too. A great improvement of caching performance can be observed compared to the traditional terrestrial small-cell networks with ST. The optimal caching scheme works very well in the JT scenarios with the aim of maximizing the cache hit probability. While considering the requirement of transmission rate and low density of UAVs, caching sub-popular files is a better choice.

Author Contributions: Conceptualization, Y.C.; methodology, M.W., Y.C. and M.D.; software, M.W.; validation, M.W., Y.C. and M.D.; formal analysis, M.W. and Y.C.; writing-original draft preparation, M.W.; writing-review and editing, Y.C. and M.D. All authors have read and agreed to the published version of the manuscript.

Funding: This research was funded by Natural Science Foundation of China (NSFC) grant number 61801119.

Conflicts of Interest: The authors declare no conflict of interest.

\section{References}

1. Chen, Y.; Ding, M.; Li, J.; Lin, Z.; Mao, G.; Hanzo, L. Probabilistic Small-Cell Caching: Performance Analysis and Optimization. IEEE Trans. Veh. Technol. 2017, 66, 4341-4354. [CrossRef]

2. Ding, M.; Liu, H. Multi-Point Cooperative Communication Systems: Theory and Applications; SJTU: Shanghai, China; Springer: Berlin/Heidelberg, Germany, 2013; [CrossRef] 
3. Ao, W.C.; Psounis, K. Fast Content Delivery via Distributed Caching and Small Cell Cooperation. IEEE Trans. Mob. Comput. 2018, 17, 1048-1061. [CrossRef]

4. Chen, Z.; Lee, J.; Quek, T.Q.S.; Kountouris, M. Cooperative Caching and Transmission Design in Cluster-Centric Small Cell Networks. IEEE Trans. Wirel. Commun. 2017, 16, 3401-3415. [CrossRef]

5. Zhou, F.; Fan, L.; Wang, N.; Luo, G.; Tang, J.; Chen, W. A Cache-Aided Communication Scheme for Downlink Coordinated Multipoint Transmission. IEEE Access 2018, 6, 1416-1427. [CrossRef]

6. Lin, P.; Song, Q.; Jamalipour, A. Multidimensional Cooperative Caching in CoMP-Integrated Ultra-Dense Cellular Networks. IEEE Trans. Wirel. Commun. 2020, 19, 1977-1989. [CrossRef]

7. Han, W.; Liu, A.; Lau, V.K.N. Dual-Mode User-Centric Open-Loop Cooperative Caching for Backhaul-Limited Small-Cell Wireless Networks. IEEE Trans. Wirel. Commun. 2019, 18, 532-545. [CrossRef]

8. Wang, L.; Wong, K.; Jin, S.; Zheng, G.; Heath, R.W. A New Look at Physical Layer Security, Caching, and Wireless Energy Harvesting for Heterogeneous Ultra-Dense Networks. IEEE Commun. Mag. 2018, 56, 49-55. [CrossRef]

9. Cao, X.; Yang, P.; Alzenad, M.; Xi, X.; Wu, D.; Yanikomeroglu, H. Airborne Communication Networks: A Survey. IEEE J. Sel. Areas Commun. 2018, 36, 1907-1926. [CrossRef]

10. Zhao, N.; Yu, F.R.; Fan, L.; Chen, Y.; Tang, J.; Nallanathan, A.; Leung, V.C.M. Caching Unmanned Aerial Vehicle-Enabled Small-Cell Networks: Employing Energy-Efficient Methods That Store and Retrieve Popular Content. IEEE Veh. Technol. Mag. 2019, 14, 71-79. [CrossRef]

11. Chen, M.; Mozaffari, M.; Saad, W.; Yin, C.; Debbah, M.; Hong, C.S. Caching in the Sky: Proactive Deployment of Cache-Enabled Unmanned Aerial Vehicles for Optimized Quality-of-Experience. IEEE J. Sel. Areas Commun. 2017, 35, 1046-1061. [CrossRef]

12. Wei, M.; Chen, Y.; Zhang, B.; Hu, J.; Zheng, H. The Improvement of Caching Performance by Employing MIMO on UAVs. In Proceedings of the 2020 Cross Strait Radio Science Wireless Technology Conference (CSRSWTC), Fuzhou, China, 13-16 December 2020; pp. 1-3. [CrossRef]

13. Khoshkholgh, M.G.; Navaie, K.; Yanikomeroglu, H.; Leung, V.C.M.; Shin, K.G. Randomized Caching in Cooperative UAV-Enabled Fog-RAN. In Proceedings of the 2019 IEEE Wireless Communications and Networking Conference (WCNC), Marrakesh, Morocco, 15-18 April 2019; pp. 1-6. [CrossRef]

14. Dinh, P.; Nguyen, T.M.; Assi, C.; Ajib, W. Joint Beamforming and Location Optimization for Cooperative Content-Aware UAVs. In Proceedings of the 2019 IEEE Wireless Communications and Networking Conference (WCNC), Marrakesh, Morocco, 15-18 April 2019; pp. 1-7. [CrossRef]

15. Zhou, F.; Wang, N.; Luo, G.; Fan, L.; Chen, W. Edge Caching in Multi-UAV-Enabled Radio Access Networks: 3D Modeling and Spectral Efficiency Optimization. IEEE Trans. Signal Inf. Process. Netw. 2020, 6, 329-341. doi:10.1109/TSIPN.2020.2986360. [CrossRef]

16. Lin, X.; Xia, J.; Wang, Z. Probabilistic caching placement in UAV-assisted heterogeneous wireless networks. Phys. Commun. 2019, 33, 54-61. [CrossRef]

17. Lyu, J.; Zeng, Y.; Zhang, R. UAV-Aided Offloading for Cellular Hotspot. IEEE Trans. Wirel. Commun. 2018, 17, 3988-4001. [CrossRef]

18. Ji, L.; Chen, J.; Feng, Z. Spectrum allocation and performance analysis for backhauling of UAV assisted cellular network. China Commun. 2019, 16, 83-92. [CrossRef]

19. Ali, M.A.; Jamalipour, A. UAV-Aided Cellular Operation by User Offloading. IEEE Internet Things J. 2020, doi:10.1109/JIOT.2020. 3015479. [CrossRef]

20. Ji, J.; Zhu, K.; Niyato, D.; Wang, R. Joint Cache Placement, Flight Trajectory, and Transmission Power Optimization for Multi-UAV Assisted Wireless Networks. IEEE Trans. Wirel. Commun. 2020, 19, 5389-5403. [CrossRef]

21. Fan, C.; Zhang, T.; Zeng, Z. Coverage and Rate Analysis of Cache-Enabled Vertical Heterogeneous Networks. IEEE Access 2019, 7, 153519-153532. [CrossRef]

22. Lakiotakis, E.; Sermpezis, P.; Dimitropoulos, X. Joint Optimization of UAV Placement and Caching under Battery Constraints in UAV-Aided Small-Cell Networks. In Proceedings of the ACM SIGCOMM 2019 Workshop on Mobile AirGround Edge Computing, Systems, Networks, and Applications, Beijing, China, 19-23 August 2019; pp. 8-14. [CrossRef]

23. Wu, H.; Tao, X.; Zhang, N.; Shen, X. Cooperative UAV Cluster-Assisted Terrestrial Cellular Networks for Ubiquitous Coverage. IEEE J. Sel. Areas Commun. 2018, 36, 2045-2058. [CrossRef]

24. Khuwaja, A.A.; Zhu, Y.; Zheng, G.; Chen, Y.; Liu, W. Performance Analysis of Hybrid UAV Networks for Probabilistic Content Caching. IEEE Syst. J. 2020, 1-12. [CrossRef]

25. Zhang, Y.; Cai, Y.; Zhang, J. Downlink Coverage Performance Analysis of UAV Assisted Terrestrial Cellular Networks. In Proceedings of the 2019 IEEE 19th International Conference on Communication Technology (ICCT), Xi'an, China, 16-19 October 2019; pp. 551-555. [CrossRef]

26. Meng, Z.; Chen, Y.; Ding, M.; López-Pérez, D. A New Look at UAV Channel Modeling: A Long Tail of LoS Probability. In Proceedings of the 2019 IEEE 30th Annual International Symposium on Personal, Indoor and Mobile Radio Communications (PIMRC), Istanbul, Turkey, 8-11 September 2019; pp. 1-6. [CrossRef]

27. Fotouhi, A.; Ding, M.; Hassan, M. Service on Demand: Drone Base Stations Cruising in the Cellular Network. In Proceedings of the 2017 IEEE Globecom Workshops (GC Wkshps), Singapore, 4-8 December 2017; pp. 1-6. [CrossRef] 
28. 3GPP. Further Advancements for E-UTRA Physical Layer Aspects. TR 36.814 (V9.0.0). 2010. Available online: http://www.doc8 8.com/p-7952437319214.html (accessed on 1 April 2021).

29. Shi, L.; Li, Z.; Bi, X.; Liao, L.; Xu, J. Full-Duplex Multi-Hop Wireless Networks Optimization with Successive Interference Cancellation. Sensors 2018, 18, 4301. [CrossRef] [PubMed]

30. Chen, Y.; Ding, M.; López-Pérez, D. Performance of Ultra-Dense Networks With a Generalized Multipath Fading. IEEE Wirel. Commun. Lett. 2019, 8, 1419-1422. [CrossRef]

31. Gradshteyn, I.; Ryzhik, I. Table of Integrals, Series and Products; Academic Press: Cambridge, MA, USA, 2007; [CrossRef]

32. Chen, Y.; Ding, M.; López-Pérez, D.; Lin, Z.; Mao, G. A Space-Time Analysis of LTE and Wi-Fi Inter-Working. IEEE J. Sel. Areas Commun. 2016, 34, 2981-2998. [CrossRef]

33. Wen, W.; Cui, Y.; Zheng, F.; Jin, S.; Jiang, Y. Random Caching Based Cooperative Transmission in Heterogeneous Wireless Networks. IEEE Trans. Commun. 2018, 66, 2809-2825. [CrossRef]

34. Kim, S.A.; An, D.G.; Ryu, H.; Kim, J. Efficient SNR estimation in OFDM system. In Proceedings of the 2011 IEEE Radio and Wireless Symposium, Phoenix, AZ, USA, 16-19 January 2011; pp. 182-185. [CrossRef] 